\title{
A Microwave Signal Generation Technique Based on Brillouin-Erbium Fiber Laser
}

\author{
N. A. B. Ahmad ${ }^{1, *}$, S. H. Dahlan', M. H. Jamaluddin ${ }^{2}$, R. Sanchez-Lara ${ }^{3}$, N. A. \\ Cholan ${ }^{1}$
}

${ }^{1}$ Faculty of Electrical and Electronic Engineering, Universiti Tun Hussein Onn Malaysia (UTHM), Parit Raja, 86400, Johor, MALAYSIA

${ }^{2}$ Wireless Communication Centre, School of Electrical Engineering, Universiti Teknologi Malaysia (UTM), Skudai, 81310, Johor Bharu, MALAYSIA

${ }^{3}$ Universidad Autónoma del Carmen, Facultad de Ingeniería, C. 56 No. 4, C.P. 24180, Cd. Del Carmen, Campeche MEXICO

*Corresponding Author

DOI: https://doi.org/10.30880/ijie.2019.11.01.005

Received 09 April 2018; Accepted 18 November 2018; Available online 30 April 2019

\begin{abstract}
An all-optical microwave signal generator based on multiwavelength Brillouin-Erbium fiber laser (MBEFL) is proposed. The MBEFL unit is designed at fixed wavelength spacing of $0.084 \mathrm{~nm}$, which corresponds to $\sim 10 \mathrm{GHz}$ carrier signal. The underlying mechanism MBEFL unit is by recycling the backscattered Stokes waves into the cavity to generate higher-order Stokes waves. Heterodyning process is then applied to the Brillouin pump (BP) consisted of first-order Brillouin Stokes (BS1) (0.084 nm spacing) and second-order Brillouin Stokes (BS2) $(0.168 \mathrm{~nm}$ spacing) signals by using a photodetector (PD). The heterodyned outputs are microwave signals at the frequencies of $10.56 \mathrm{GHz}$ and $21.2 \mathrm{GHz}$, relative to first order and second order Stokes waves, respectively. These microwave signals are experimentally achieved by controlling the EDF pump power where the EDF pump power can be as low as $20 \mathrm{~mW}$.
\end{abstract}

Keywords: Microwave carrier, stimulated Brillouin scattering, fiber laser

\section{Introduction}

Generally, photonic technology provides substantial advantages over conventional electronics, namely low loss and large bandwidth of the state-of-the-art optical fibers, and immunity to electromagnetic interferences [1]. Since the range of applications of telecommunications are growing aggressively, the requirement for higher frequency spectrum is largely in demand now, especially for the case of $5^{\text {th }}$ generation $(5 \mathrm{G})$ applications [2]. Therefore, tremendous efforts into developing signal generators to generate both microwave and millimeter waves by using photonic techniques can be observed [3]. Some of the widely knows methods are Optical injection locking (OIL), Optical Phase-Lock Loop (OPLL), external modulation, and fiber lasers [4]. However, recently stimulated Brillouin scattering (SBS) based signal generator had received wide attention [5,6]. The SBS is selected as the gain mechanism because of its relatively low nonlinear threshold, offers great stability as a signal generator, and narrow linewidth [7]. A straightforward method to generate microwave signals is by using optical heterodyne detection with either two independent lasers with required wavelength spacing or dual-wavelength based laser [8]. Generating signals above $11 \mathrm{GHz}$ requires multiwavelength laser generation, whereas only two lasing lines will be selected for the heterodyning process at the photodiode [9]. In 
order to address this requirement, the Brillouin fiber lasers (BFL) with narrow gain profiles are excellent candidates to enhance the number of Stokes lines [10], which had been used as microwave signal generator as reported in [11].

Within the BFL technology, there are many reported methods in selecting two lasing lines in accordance with the largest spacing. In [12], a double Brillouin Stokes shifter (DBS) scheme was employed, whereby heterodyning between the initial Brillouin pump (BP) with the second-order Brillouin Stokes (BS2), fourth-order Brillouin Stokes (BS4), and six order Brillouin Stokes (BS6) resulting wavelength spacing of $0.178 \mathrm{~nm}, 0.34 \mathrm{~nm}$, and $0.52 \mathrm{~nm}$ respectively. Alternatively, in [13], a dual laser source was replaced by incorporating BFL and fiber Bragg gratings (FBGs). However, the two FBGs need to control precisely in order to assure that both had the same center wavelength.

Therefore, the present art aims to propose and demonstrate experimentally a simple, efficient, and robust development of an all optical microwave signal generator. The proposed method applies optical couplers and circulator to replace the FBG. The backscattered Stokes waves are recycled into a cavity through the port 3 of the circulator in order to generate multiwavelength Brillouin-Erbium fiber laser (MBEFL). This method applies Erbium-doped Fiber Amplifier (EDFA) to boost the Brillouin pump (BP) signal. The wavelength spacing between the pump and the Stokes wave is $0.08 \mathrm{~nm}$, which corresponds to $\sim 10 \mathrm{GHz}$. This method is capable of generating microwave signals of up to 100 $\mathrm{GHz}$ by heterodyning the initial BP with the higher-order Brillouin's Stokes waves.

\section{Experimental Setup}

The experimental setup of the MBEFL is illustrated in Fig. 1. The MBEFL unit uses recycling technique of the backscattered Stokes as the SBS enhancing factor, subsequently amplified by erbium-doped fiber (EDF) and converted to electrical signal by the photodiode (PD). An external tunable laser source (TLS) is configured to emit maximum power of $13 \mathrm{dBm}$ at $1550 \mathrm{~nm}$ is connected to this system via the $10 \%$ port of the Coupler (90/10 ratio) to provide the Brillouin pump (BP) source. An Isolator 1 was placed after the TLS to protect the device from any possible damage arising for reflected signals. Thereafter, the BP source is coupled with a $980 \mathrm{~nm}$ pump laser diode (LD) through a 980/1550 nm wavelength division multiplexing (WDM) coupler, and subsequently amplified via $10 \mathrm{~m}$ Erbium-doped fiber (EDF). The amplified signal is then launched into a $12 \mathrm{~km}$ dispersion shifted fiber (DSF), which is the Brillouin gain medium for the proposed work. In more details, the DSF made by is Lucent/AT\&T with effective group index of 1.470 and an attenuation factor of $0.22 \mathrm{~dB} / \mathrm{km}$ at $1550 \mathrm{~nm}$, effective cross-sectional area of $86.6 \mu \mathrm{m}^{2}$, and nonlinear coefficient of $2.8 \mathrm{~W}^{1} \mathrm{~km}^{-1}$. At the output of the DSF, the first-order Brillouin Stokes (BS1) is generated when the BP power exceeds the SBS threshold of the gain medium. The BS1 from the DSF is connected to an optical circulator, where the signal is recirculated back to the cavity via Port 3, and subsequently propagates through the port-90\% of the 90/10 coupler.

Essentially a ring-like laser is formed. Due to the recirculating process via 90/10 coupler, the continuously emitting $\mathrm{BP}$ and newly formed BS1 coupled together and jointly being amplified, and consequently channeled back to the $12 \mathrm{~km}$ DSF for the second-order Brillouin Stokes (BS2) generation. For the generation of any subsequent higher-order Brillouin Stokes signals, the same mechanism will be applied within the MBEFL system. The BP with BS1 and the BP with BS2 are then mixed at the PD (50-GHz dual-window PD) and converted to microwave signal. At the output of the PD, a spectrum analyzer (ESA) (E4407B, Agilent) is connected for signal analysis. An Isolator 2 is connected between DSF and PD to ensure that the output of the laser will not interfere with the Fresnel reflections to achieve a highly stable lasing condition.

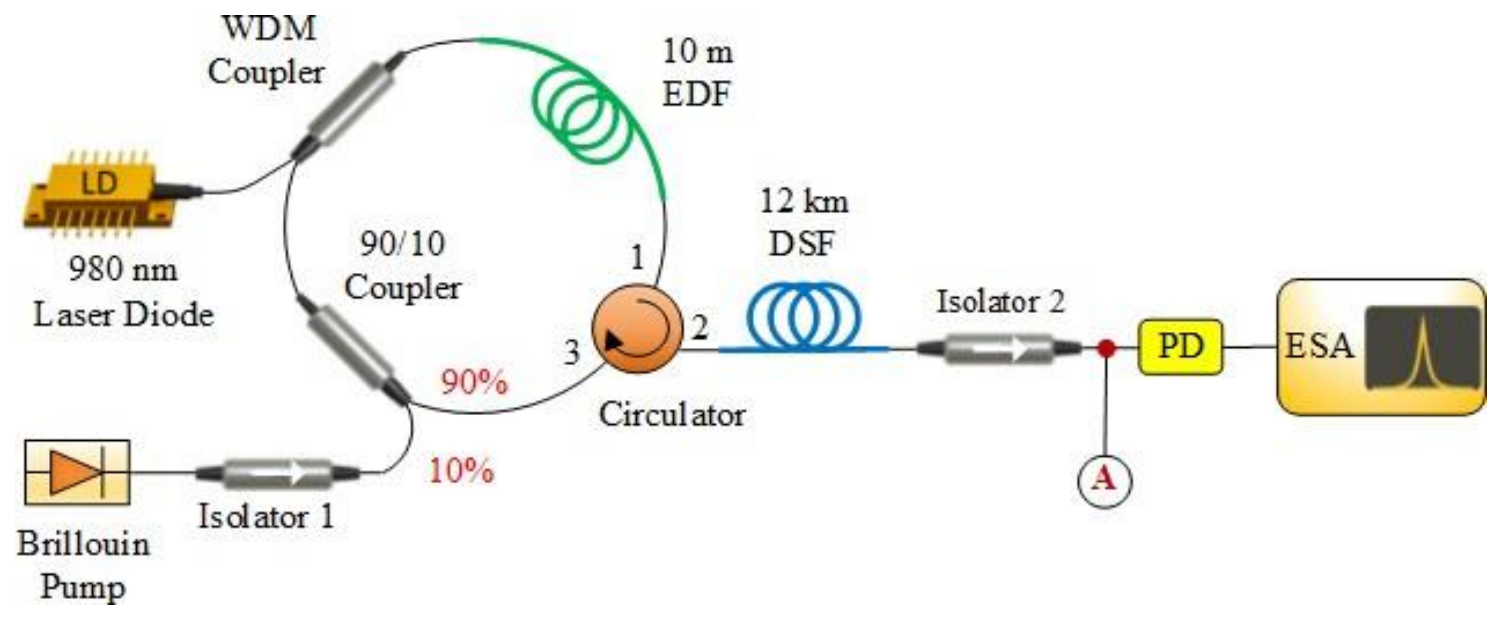

Fig. 1 - Experimental setup for the all-optical microwave signal generator. 


\section{Results and Discussion}

The output at port A (see Fig. 1) is connected to an optical spectrum analyzer (OSA) (AQ6370B, Yokogawa) with a resolution of $0.02 \mathrm{~nm}$. The corresponding output spectrum of the two different operation states of the MBEFL was measured by OSA. At Brillouin pump (BP) power of $5 \mathrm{dBm}$, and the EDF pump power configured at $20 \mathrm{~mW}$, the first order Brillouin Stokes (BS1) started to occur, as shown in Fig. 2 (a). The measured BP and BS1 wavelengths are $1550.116 \mathrm{~nm}$ and $1550.2 \mathrm{~nm}$, respectively. It is clear now that the system requires very low EDF pump power to generate the first order Stokes line. The generated BS1 is then channeled to the PD. In this case, the wavelength spacing between the BP and BS1 is measured at $0.084 \mathrm{~nm}$.

Upon increasing the EDF pump power to $25 \mathrm{~mW}$, the BS2 will be initialized, as shown in Fig. 2 (b). BS1 maintains its existence but increased in amplitude. The measured wavelengths of BP, BS1, and BS2 are $1550.116 \mathrm{~nm}$, $1550.2 \mathrm{~nm}$, and $1550.284 \mathrm{~nm}$ respectively. The wavelength spacing between the BP and BS2 is measured at $0.168 \mathrm{~nm}$. Based on the results obtained, it is clear that higher order Brillouin Stokes (BS3, BS4 ... and so on) can be generated by simply increasing the EDF pump power, where the consequent newly occurring Stokes lines will have a similar wavelength difference of $0.084 \mathrm{~nm}$ with its adjacent existing Stokes wave. Thus, this fixed wavelength difference between adjacent Stokes waves can be translated into a predictable frequency values upon converting into microwave signals.

The generated microwave signals as a function of varying EDF pump powers of $20 \mathrm{~mW}$ and $25 \mathrm{~mW}$ are depicted in Fig. 3. The microwave signal peak in Fig. 3 (a) corresponds to the BP beating with BS1 (see Fig. 2 (a) for optical domain). The results exhibited that the generated microwave signal has a frequency and peak power of $10.56 \mathrm{GHz}$ and $20 \mathrm{dBm}$, respectively. In Fig. 3 (b), the microwave signal is generated at $21.2 \mathrm{GHz}$ with $-24 \mathrm{dBm}$ peak power, which is a result of heterodyne between BP and BS2 (Fig. 2 (b)).
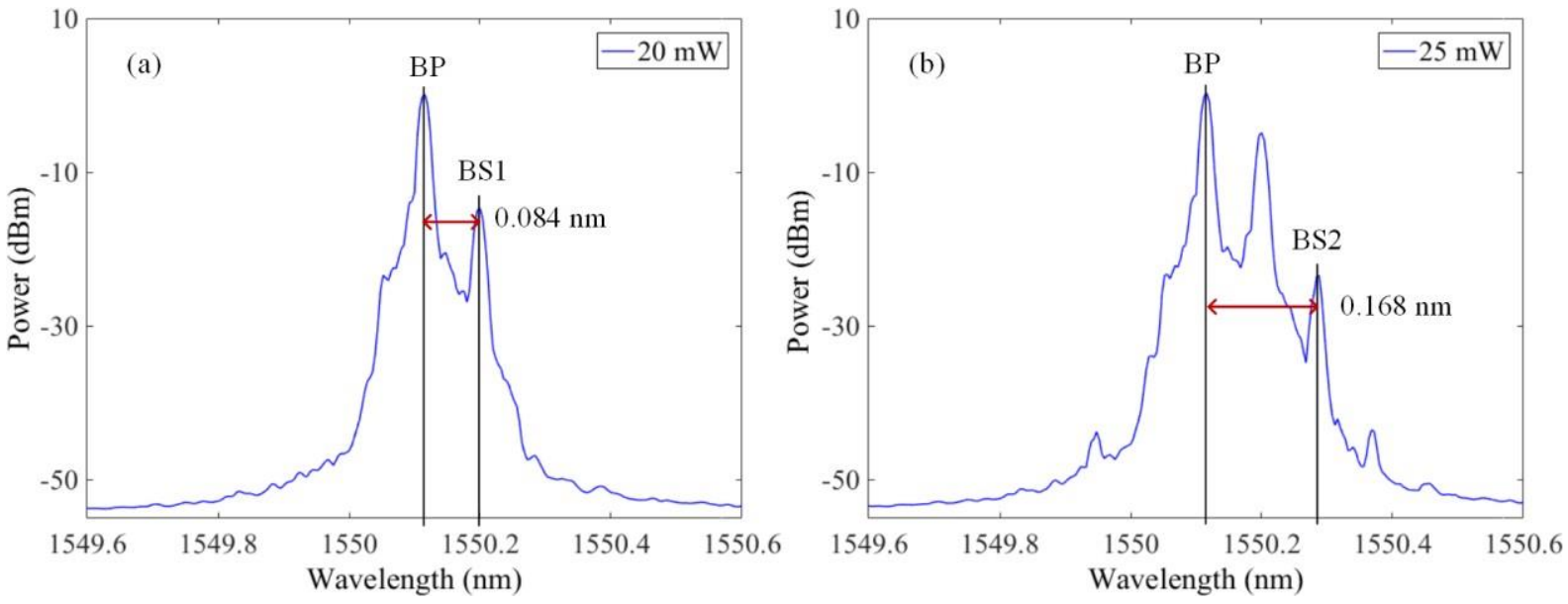

Fig. 2 - Optical spectrum due to EDF pump power at (a) $20 \mathrm{~mW}$ and (b) $25 \mathrm{~mW}$.
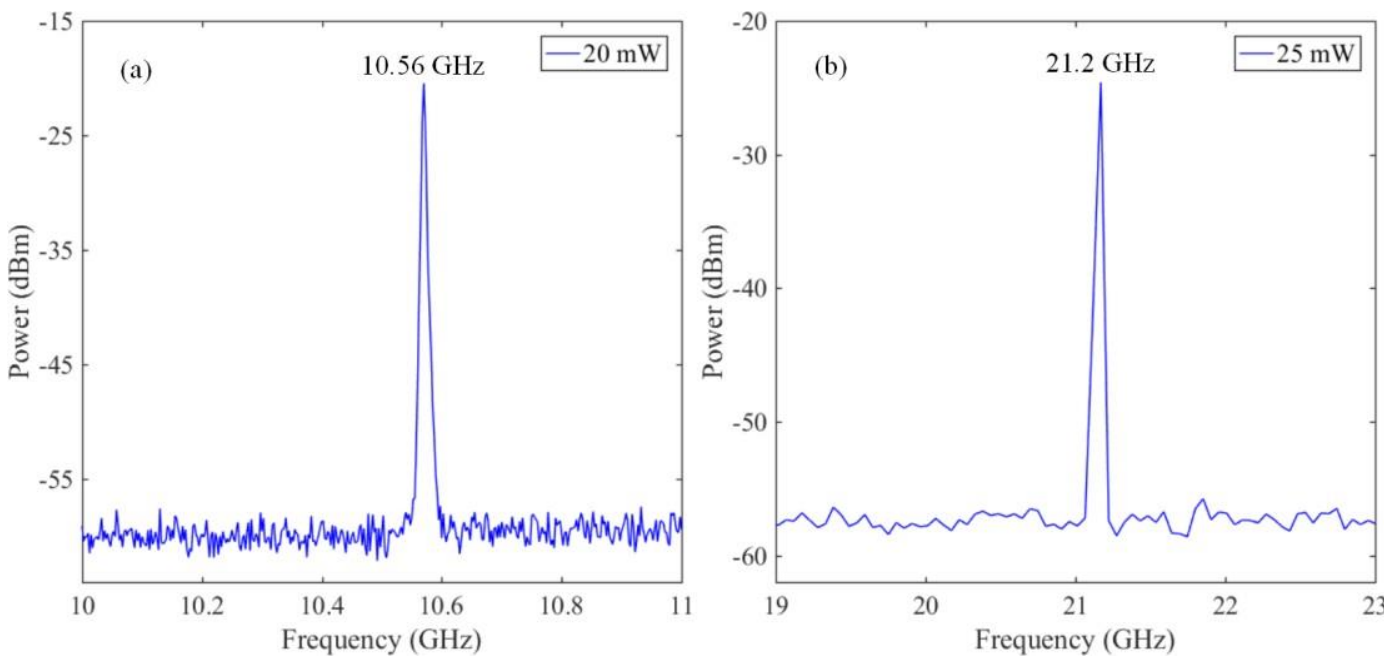

Fig. 3 - Frequency spectrum measured at the output of photodetector, where (a) BP BS1 beating process and, (b) BP and BS2 beating process. 
The beating frequency between the BP and the BS1/BS2 are predictable, and can be governed by using the electromagnetic wave coupling equations [14]

$$
\begin{aligned}
& E_{1}(t)=E_{01} \cos \left(\omega_{1} t+\varphi_{1}\right) \\
& E_{2}(t)=E_{02} \cos \left(\omega_{2} t+\varphi_{2}\right)
\end{aligned}
$$

where $E_{01}, \omega_{1}$, and $\varphi_{1}$ are the amplitude, angular frequency, and phase of the initial BP, respectively. While, $E_{02}, \omega_{2}$, and, $\varphi_{2}$ are the amplitude, angular frequency, and phase of the higher-order Brillouin Stokes wave. The output current at the photodetector can be computed by:

$$
I_{R F}=A \cos \left[\left(\omega_{1}-\omega_{2}\right) t+\left(\varphi_{1}-\varphi_{2}\right)\right]
$$

Equation (3) denotes the advantages of SBS-based microwave signal generation where the power of the microwave signal can be controlled directly by adjusting the peak power of the higher-order Brillouin Stokes wave.

\section{End-to-end Wireless Transmission}

As a proof of concept, we built a test-bed to perform actual signal transmission between two horn antennas that connected to MBEFL system (Fig. 4). In this experiment, the MBEFL system is connected to a horn antenna and acted as the transmitter system. Another horn antenna is connected to a spectrum analyzer and hence acted as receiver. Both horn antennas are from BHA 9118 model with the frequency range from $1 \mathrm{GHz}$ to $18 \mathrm{GHz}$. These antennas are separated at a distance of $\mathrm{R}=1 \mathrm{~m}$. The directivity is $11.6 \mathrm{dBi}$ at $f=10.56 \mathrm{GHz}$ (as provided by the manufacturer). The output power of the transmitter is configured at $P_{T}=-20 \mathrm{dBm}$. As a result, the measured receive power, $P_{R}$ is $-50 \mathrm{dBm}$, as shown in Fig. 5. The output measurement agrees with the approximation made by Equation (4).

$$
\begin{aligned}
P_{R}(d B m) & =P_{T}(d B m)+D_{R}(d B i)+D_{T}(d B i)+\left|\frac{(\lambda)^{2}}{(4 \pi R}\right|^{2} \\
& =-20+11.6+11.6+(-52.86) \\
& =-49.66
\end{aligned}
$$

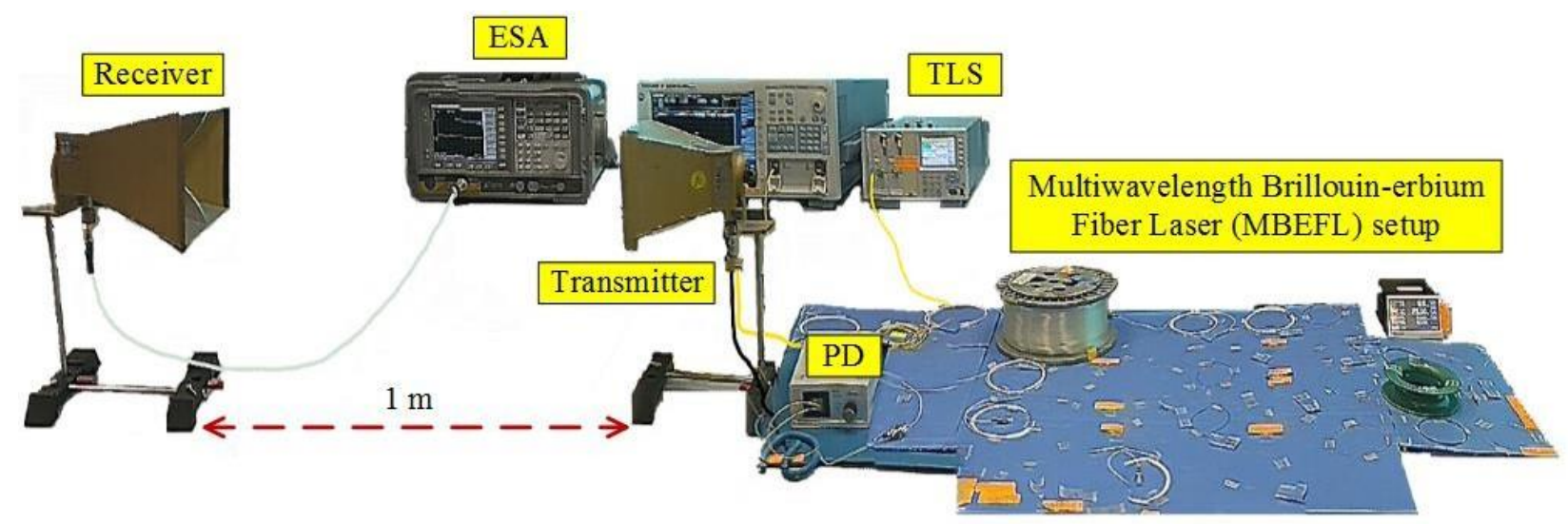

Fig. 4 - Experimental setup for signal transmission with MBEFL system. 


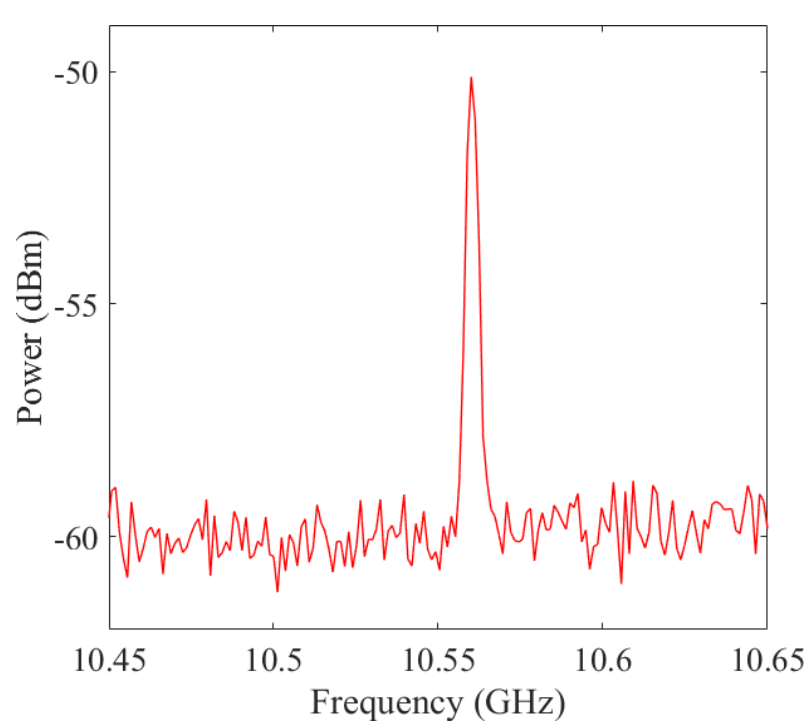

Fig. 5 - Measured spectrum at the receiver.

\section{Conclusion}

In this paper, a technique to generate microwave signal based on photonic technology is discussed. The method to generate microwave signal proposed in this paper presents simplicity and straightforward implementation compared to previous works as it does not require microwave reference sources [15]. The results show that this MBEFL are capable to generate frequency of $10.56 \mathrm{GHz}$ and $21.2 \mathrm{GHz}$ with the peak power of $-20 \mathrm{dBm}$ and $-24 \mathrm{dBm}$, respectively. In principle, this technique can be used to produce microwave signals of up to hundreds of gigahertz. However, the capability is limited to photodiode's (PD) limitations.

\section{Acknowledgement}

This work was supported in part by Fundamental Research Grant Scheme (FRGS) Vot 1622 by the Ministry of Higher Education (MOHE) Malaysia.

\section{References}

[1] Ye, Q., Gao, Y., \& Yang, C. (2016). A novel phase noise measurement of phase modulation microwave photonic links. Journal of Modern Optics, 63, 1270-1273.

[2] Hu, J., Gu, Y., Tan, W, Zhu, W, Wang, L., \& Zhao, M. (2016). Microwave photonic link with improved phase noise using a balanced detection scheme. Optics Communications, 370, 1-5.

[3] Capmany, J., \& Novak, D. (2007). Microwave photonics combines two worlds. Nature Photonics, 1, 319-330.

[4] Yao, J. (2009). Microwave photonics. Journal of Lightwave Technology, 27, 314-335.

[5] Kanesan, T., Maskuriy, F., Hafiz, M., Mohamad, R., Mitani, S. M., Hizan, H. M., Rahim, A. I. A., Haigh, P. A., Rajbhandari, S., \& Chang, G. K. (2015). Dual pump Brillouin laser for RoF millimeter-wave carrier generation with tunable resolution. IEEE Region 10 Annual International Conference, Proceedings (TENCON), 2016, 1-6.

[6] Al-Dabbagh, R. K., \& Al-Raweshidy, H. S. (2016). Photonic methods of millimeter-wave generation based on Brillouin fiber laser. Optics and Laser Technology, 79, 124-131.

[7] Danion, G., Frein, L., Bacquet, D., Pillet, G., Molin, S., Morvan, L., Ducournao, G., Vallet, M., Szriftgiser, P., \& Louini, M. (2016). Mode-Hopping suppression in long Brillouin fiber laser with non-resonant pumping. Optics Letters, 41, 2362-2365.

[8] Sun, T., Guo, Y., Wang, T., Huo, J., \& Zhang, L. (2015). Dual-wavelength single longitudinal mode fiber laser for microwave generation. Optics and Laser Technology, 67, 143-145.

[9] Liu, Y., Zhang, M., Wang, P., Li, L., Wang, Y., \& Bao, X. (2015). Multiwavelength single-longitudinal-mode Brillouin-erbium fiber laser sensor for temperature measurements with ultrahigh resolution. IEEE Photonics Journal, 7, 7182751.

[10] Ahmad, N. A. B., Dahlan, S. H., \& Cholan, N. A. (2016). Experimental investigation of the residual waves in a multiwavelength Brillouin-erbium fiber laser. 2016 IEEE $6^{\text {th }}$ International Conference on Photonics (ICP 2016), 7510021. 
[11] Xiang, D., Lu, P., Xu, Y., Chen, L., \& Bao, X. (2016). Random Brillouin fiber laser for tunable ultra-narrow linewidth microwave generation. Optics Letters, 41, 4839-4842.

[12] Mohamad, R., Idrus, S. M., Supaat, A. S., Yaakob, S., Zamzuri, A. K., \& Sukito, S. N. A. (2014). Millimeter wave carrier generation based on Brillouin fiber laser with improved tuning capability. Optik, 125, $205-207$.

[13] Shee, Y. G., Al-Mansoori, M. H., Yaakob, S., Man, A., Zamzuri, K., Adikan, F. R. M., \& Mahdi, M. A. (2012). Millimeter wave carrier generation based on a double-Brillouin-frequency spaced fiber laser. Optics Express, 20, 13402-13408.

[14] Prasad, K. R. Y., Srinivas, T., \& Ramana, D. V. (2015). Design and demonstration of hardware efficient OPLL for generation of stable microwave signals by optical heterodyning. Journal of Optics (India), 44, 103-118.

[15] Nanzer, J. A, Callahan, P. T, Dennis, M. L., Clark Jr., T. R., Novak, D., \& Waterhouse, R. B. (2011). Millimeterwave wireless communication using dual-wavelength photonic signal generation and photonic upconversion. IEEE Transactions on Microwave Theory and Techniques, 59, 3522-3530. 